\title{
HOMOGENEOUS RICCI SOLITON HYPERSURFACES IN THE COMPLEX HYPERBOLIC SPACES
}

\author{
TAKAHIRO HASHINAGA, AKIRA KUBO, AND HIROSHI TAMARU \\ Abstract. A Lie hypersurface in the complex hyperbolic space is an orbit \\ of a cohomogeneity one action without singular orbit. In this paper, we \\ classify Ricci soliton Lie hypersurfaces in the complex hyperbolic spaces.
}

\section{INTRODUCTION}

Homogeneous submanifolds in Riemannian symmetric spaces of noncompact type $G / K$ have provided a lot of interesting examples of submanifolds. We refer to [1, 3, 4, 15, 6, 8, 9, 10, 16, and references therein. Typical and the simplest examples are given by the nilpotent part $N$ of the Iwasawa decomposition $G=$ $K A N$. All orbits of $N$ in $G / K$ are isometrically congruent to each other (see [3, 10]), and they are known to be Ricci solitons ([12]). Recall that a complete Riemannian manifold $(M, g)$ is called a Ricci soliton if

$$
\operatorname{Ric}_{g}=c g-\frac{1}{2} \mathcal{L}_{X} g
$$

holds for some $c \in \mathbb{R}$ and some complete vector field $X$ on $M$, where $\mathrm{Ric}_{g}$ denotes the Ricci operator of $(M, g)$ and $\mathcal{L}_{X}$ is the usual Lie derivative. The vector field $X$ is called the potential vector field. A Ricci soliton is a natural generalization of an Einstein manifold.

In symmetric spaces of noncompact type, homogeneous hypersurfaces called Lie hypersurfaces are of particular interest. For an isometric action on a Riemannian manifold, maximal dimensional orbits are said to be regular, and other orbits singular. The codimension of a regular orbit is called the cohomogeneity of an action. A Lie hypersurface is an orbit of a cohomogeneity one action without singular orbit. The notion of Lie hypersurfaces has been introduced by Berndt ([1]). For symmetric spaces of noncompact type $G / K$, the orbits of a cohomogeneity one action without singular orbit form a Riemannian foliation ([2]). Furthermore, when $G / K$ is irreducible, such actions have been completely classified up to orbit equivalence ([4]). Recall that two isometric actions on a Riemannian manifold are said to be orbit equivalent if there exists an isometry of the manifold mapping the orbits of one of these actions onto the orbits of the other action.

Key words and phrases. Real hypersurfaces, homogeneous submanifolds, complex hyperbolic spaces, Ricci solitons.

2010 Mathematics Subject Classification. Primary 53C40, Secondary 53C30, 53C25, 53C35.

The first author was supported in part by Grant-in-Aid for JSPS Fellows (11J05284). The third author was supported in part by KAKENHI (24654012). 
In this paper, we focus on Lie hypersurfaces in the complex hyperbolic spaces

$$
\mathbb{C H}^{n}=\mathrm{SU}(1, n) / \mathrm{S}(\mathrm{U}(1) \times \mathrm{U}(n))
$$

with $n \geq 2$. Owing to [4], for rank one symmetric spaces of noncompact type, there exist exactly two cohomogeneity one actions without singular orbit up to orbit equivalence. One of the actions is given by $N$, the nilpotent part of the Iwasawa decomposition of the isometry group. The orbits of $N$, which are isometrically congruent to each other, are horospheres. The other action induces the so-called solvable foliation. In the case of the real hyperbolic space $\mathbb{R} H^{n}$, intrinsic geometry of the Lie hypersurfaces is well-known. In fact, the horosphere is flat, and the solvable foliation consists of a totally geodesic $\mathbb{R} H^{n-1}$ and its equidistant hypersurfaces. It is easy to see that all Lie hypersurfaces in $\mathbb{R H}^{n}$ have constant curvature. On the contrary, in the case of $\mathbb{C H}^{n}$ with $n \geq 2$, the situation is much more interesting and nontrivial. In this case, the solvable foliation consists of the homogeneous ruled minimal hypersurface and its equidistant hypersurfaces. The homogeneous ruled minimal hypersurface in $\mathbb{C H}^{n}$ is the ruled real hypersurface determined by a horocycle in a totally geodesic $\mathbb{R H}^{2}$ in $\mathbb{C H}^{n}([14])$. Note that this is isometric to a fan (we refer to [11).

The purpose of this paper is to study and classify Ricci soliton Lie hypersurfaces in $\mathbb{C H}^{n}$. It is well-known that there exist no Einstein hypersurfaces in $\mathbb{C H}^{n}$ (see [15]). On the other hand, as we mentioned before, the horosphere is a Ricci soliton. Our main theorem classifies Ricci soliton Lie hypersurfaces in $\mathbb{C H}^{n}$. It would be interesting that the result is different in the cases $n=2$ and $n>2$.

Theorem. A Lie hypersurface in $\mathbb{C H}^{n}$ is a Ricci soliton if and only if

(1) it is isometrically congruent to a horosphere, or

(2) $n=2$ and it is isometrically congruent to the homogeneous ruled minimal hypersurface.

The above theorem is relevant to the results by Cho and Kimura ([7]). Among others, for real hypersurfaces in non-flat complex space forms, they proved

- there do not exist compact Hopf hypersurfaces which are Ricci solitons,

- there do not exist ruled hypersurfaces which are gradient Ricci solitons.

Recall that a Ricci soliton is said to be gradient if the potential vector field can be expressed as the gradient of a smooth function. We also recall that a hypersurface in $\mathbb{C H}^{n}$ is said to be Hopf if the structure vector field $J \xi$ is an eigenvector of the shape operator, where $J$ is the complex structure and $\xi$ is a unit normal vector. Our theorem implies that the assumptions on these nonexistence results cannot be removed. Note that a horosphere is a Hopf hypersurface (see [1, 9]), which is a Ricci soliton, but not compact. When $n=2$, the homogeneous ruled minimal hypersurface is a ruled hypersurface, which is a Ricci soliton, but not gradient (see [13]).

This paper is organized as follows. In Section 2, we recall algebraic Ricci solitons, which play important roles in studying homogeneous Ricci solitons. In Section 3, we recall some necessary results on Lie hypersurfaces in $\mathbb{C H}^{n}$. In Section 4 we prove the main theorem. 


\section{Ricci solitons And Algebraic RicCi solitons}

The notion of algebraic Ricci solitons has essentially been introduced by Lauret $([12,13])$. In this section, we recall a relationship between left-invariant Ricci solitons and algebraic Ricci solitons. Let $G$ be a simply-connected Lie group, and $g$ be a left-invariant metric on $G$. The corresponding metric Lie algebra of $(G, g)$ is a pair $(\mathfrak{g},\langle\rangle$,$) , where \mathfrak{g}$ is the Lie algebra of $G$ and $\langle$,$\rangle is the$ inner product on $\mathfrak{g}$ corresponding to $g$. We can study curvatures of $(G, g)$ in term of the corresponding metric Lie algebra $(\mathfrak{g},\langle\rangle$,$) .$

First of all, we recall some curvature formulas for a metric Lie algebra $(\mathfrak{g},\langle\rangle$,$) .$ Let $X, Y \in \mathfrak{g}$. The Levi-Civita connection $\nabla$ of $(\mathfrak{g},\langle\rangle$,$) is given by$

$$
2\left\langle\nabla_{X} Y, Z\right\rangle=\langle[X, Y], Z\rangle+\langle[Z, X], Y\rangle+\langle X,[Z, Y]\rangle \quad(Z \in \mathfrak{g}) .
$$

Then the Riemannian curvature $R$ of $(\mathfrak{g},\langle\rangle$,$) is defined by$

$$
R(X, Y):=\nabla_{[X, Y]}-\nabla_{X} \nabla_{Y}+\nabla_{Y} \nabla_{X}
$$

and the Ricci operator Ric of $(\mathfrak{g},\langle\rangle$,$) is defined by$

$$
\operatorname{Ric}(X):=\sum R\left(E_{i}, X\right) E_{i}
$$

where $\left\{E_{i}\right\}$ is an orthonormal basis of $(\mathfrak{g},\langle\rangle$,$) .$

We now recall the notion of algebraic Ricci solitons. For a Lie algebra $\mathfrak{g}$, let us denote the Lie algebra of derivations by

$$
\operatorname{Der}(\mathfrak{g}):=\{D \in \mathfrak{g l}(\mathfrak{g}) \mid D[X, Y]=[D(X), Y]+[X, D(Y)](\forall X, Y \in \mathfrak{g})\} .
$$

Definition 2.1. A metric Lie algebra $(\mathfrak{g},\langle\rangle$,$) is called an algebraic Ricci soliton$ if the following holds:

$$
\operatorname{Ric}=c \cdot \mathrm{id}+D,
$$

for some $c \in \mathbb{R}$ and $D \in \operatorname{Der}(\mathfrak{g})$.

A relationship between left-invariant Ricci solitons and algebraic Ricci solitons is given as follows. According to [13], if the corresponding metric Lie algebra $(\mathfrak{g},\langle\rangle$,$) is an algebraic Ricci soliton, then (G, g)$ is a Ricci soliton. The converse also holds if $G$ is completely solvable. Recall that $G$ is said to be completely solvable if $G$ is solvable and the eigenvalues of any ad $X$ are all real.

Theorem 2.2 ([13]). Let $(G, g)$ be a simply-connected completely solvable Lie group equipped with a left-invariant metric, and $(\mathfrak{g},\langle\rangle$,$) be the corresponding$ metric Lie algebra. Then $(G, g)$ is a Ricci soliton if and only if $(\mathfrak{g},\langle\rangle$,$) is an$ algebraic Ricci soliton.

\section{LiE HyPERSURFACES IN THE COMPLEX HYPERBOLIC SPACE}

In this section, we recall Lie hypersurfaces in the complex hyperbolic space $\mathbb{C H}^{n}$ and their curvature properties. We refer to [1, 4, 9].

Definition 3.1. The triple $(\mathfrak{s},\langle\rangle, J$,$) is called the solvable model of the complex$ hyperbolic space $\mathbb{C H}^{n}$ if 
(1) $\mathfrak{s}$ is a Lie algebra, and there exists a basis $\left\{A_{0}, X_{1}, Y_{1}, \ldots, X_{n-1}, Y_{n-1}, Z_{0}\right\}$ whose bracket products are given by

$$
\left[A_{0}, X_{i}\right]=(1 / 2) X_{i},\left[A_{0}, Y_{i}\right]=(1 / 2) Y_{i},\left[A_{0}, Z_{0}\right]=Z_{0},\left[X_{i}, Y_{i}\right]=Z_{0},
$$

(2) $\langle$,$\rangle is an inner product on \mathfrak{s}$ so that the above basis is orthonormal,

(3) $J$ is a complex structure on $\mathfrak{s}$ given by

$$
J\left(A_{0}\right)=Z_{0}, J\left(Z_{0}\right)=-A_{0}, J\left(X_{i}\right)=Y_{i}, J\left(Y_{i}\right)=-X_{i} .
$$

We use $\mathfrak{s}$ instead of $(\mathfrak{s},\langle\rangle, J$,$) for simplicity, and denote by S$ the corresponding simply-connected solvable Lie group with the induced left-invariant Riemannian metric and complex structure. It is known that $S$ can be identified with $\mathbb{C H}^{n}$ with holomorphic sectional curvature -1 . In fact, $S$ coincides with the solvable part of the Iwasawa decomposition of $\mathrm{SU}(1, n)$.

In the rest of the section, we recall some known results on Lie hypersurfaces in $\mathbb{C H}^{n}$. First of all, we recall the definition.

Definition 3.2. A Lie hypersurface in $\mathbb{C H}^{n}$ is an orbit of a cohomogeneity one action without singular orbits.

It is known that there exist exactly two cohomogeneity one actions on $\mathbb{C H}^{n}$ without singular orbit up to orbit equivalence.

Theorem $3.3([4])$. An isometric action on $\mathbb{C H}^{n}$ is a cohomogeneity one action without singular orbit if and only if it is orbit equivalent to one of the actions of $S(\pi / 2)$ or $S(0)$, where $S(\theta)$ is the connected Lie subgroup of $S$ with Lie algebra

$$
\mathfrak{s}(\theta):=\mathfrak{s} \ominus \mathbb{R}\left(\cos (\theta) X_{1}+\sin (\theta) A_{0}\right) .
$$

Note that $\ominus$ means the orthogonal complement with respect to $\langle$,$\rangle . By study-$ ing the orbits of these actions, one obtains the classification of Lie hypersurfaces in $\mathbb{C H}^{n}$ up to isometric congruence.

Theorem 3.4 ([1]). Every Lie hypersurface in $\mathbb{C H}^{n}$ is isometrically congruent to the orbit $S(\theta)$.o through the origin o for some $\theta \in[0, \pi / 2]$.

Note that $S(\pi / 2)=N$ and hence the orbit $S(\pi / 2) . o$ is a horosphere. The orbit $S(0) . o$ is the homogeneous ruled minimal hypersurface. If $0<\theta<\pi / 2$, then the orbit $S(\theta) . o$ is isometrically congruent to $S(0) . p$ for some $p$, which is an equidistant hypersurface to $S(0)$.o.

We now study the geometry of Lie hypersurfaces. The Lie hypersurface $S(\theta) . o$ can be studied in terms of the metric Lie algebra $(\mathfrak{s}(\theta),\langle\rangle$,$) , where this$ $\langle$,$\rangle denotes the restriction of \langle$,$\rangle to \mathfrak{s}(\theta)$. First of all, by definition, $\mathfrak{s}(\theta)$ is a codimension one subalgebra of $\mathfrak{s}$, and it is solvable. Denote by

$$
T:=\cos (\theta) A_{0}-\sin (\theta) X_{1}, \quad \mathfrak{v}_{0}:=\operatorname{span}\left\{X_{2}, Y_{2}, \ldots, X_{n-1}, Y_{n-1}\right\} .
$$

Then we have the orthogonal decomposition

$$
\mathfrak{s}(\theta)=\operatorname{span}\{T\} \oplus \operatorname{span}\left\{Y_{1}\right\} \oplus \mathfrak{v}_{0} \oplus \operatorname{span}\left\{Z_{0}\right\} .
$$

One can directly see the following equations, which will be used hereafter.

Lemma 3.5. Let $V, W \in \mathfrak{v}_{0}$. Then we have

(1) $\left[T, Y_{1}\right]=(1 / 2) \cos (\theta) Y_{1}-\sin (\theta) Z_{0}$, 
(2) $[T, V]=(1 / 2) \cos (\theta) V$,

(3) $\left[T, Z_{0}\right]=\cos (\theta) Z_{0}$,

(4) $[V, W]=\left\langle[V, W], Z_{0}\right\rangle Z_{0}$, especially, $\left[X_{k}, Y_{k}\right]=Z_{0}$ for all $k=2, \ldots, n-1$.

Finally in this subsection, we recall the formulas for Ricci curvatures of the Lie hypersurfaces. By direct calculations in terms of $(\mathfrak{s}(\theta),\langle\rangle$,$) , one has the$ following.

Proposition $3.6([9])$. The Ricci operator of $(\mathfrak{s}(\theta),\langle\rangle$,$) satisfies$

(1) $\operatorname{Ric}(T)=-(1 / 4)\left(2+(2 n-1) \cos ^{2}(\theta)\right) T$,

(2) $\operatorname{Ric}\left(Y_{1}\right)=-(1 / 4)\left(2+(2 n-3) \cos ^{2}(\theta)\right) Y_{1}+(n / 2) \sin (\theta) \cos (\theta) Z_{0}$,

(3) $\operatorname{Ric}(V)=-(1 / 4)\left(2+(2 n-1) \cos ^{2}(\theta)\right) V$ for any $V \in \mathfrak{v}_{0}$,

(4) $\operatorname{Ric}\left(Z_{0}\right)=(n / 2) \sin (\theta) \cos (\theta) Y_{1}+(1 / 2)\left((n-1)-2 n \cos ^{2}(\theta)\right) Z_{0}$.

\section{MAIN RESULT}

In this section we classify Ricci soliton Lie hypersurfaces in $\mathbb{C H}^{n}$. First of all, we see that we have only to work on algebraic Ricci solitons.

Lemma 4.1. For any $\theta \in[0, \pi / 2]$, the Lie hypersurface $S(\theta)$.o is a Ricci soliton if and only if $(\mathfrak{s}(\theta),\langle\rangle$,$) is an algebraic Ricci soliton.$

Proof. Let $\theta \in[0, \pi / 2]$. Owing to Theorem 2.2 , we have only to check that $\mathfrak{s}(\theta)$ is completely solvable. Take any $X \in \mathfrak{s}(\theta)$. One can write

$$
X=a_{1} T+a_{2} Y_{1}+V+a_{3} Z_{0} \quad\left(\text { for } V \in \mathfrak{v}_{0}\right) .
$$

According to Lemma 3.5, we can show directly that

$$
\begin{aligned}
\operatorname{ad} X(T)=- & (1 / 2) a_{2} \cos (\theta) Y_{1}-(1 / 2) \cos (\theta) V \\
& -\left(a_{2} \sin (\theta)+a_{3} \cos (\theta)\right) Z_{0}, \\
\operatorname{ad} X\left(Y_{1}\right)= & (1 / 2) a_{1} \cos (\theta) Y_{1}+a_{1} \sin (\theta) Z_{0}, \\
\text { ad } X(W)= & (1 / 2) a_{1} \cos (\theta) W+\left\langle[V, W], Z_{0}\right\rangle Z_{0}, \\
\operatorname{ad} X\left(Z_{0}\right)= & a_{1} \cos (\theta) Z_{0},
\end{aligned}
$$

where $W \in \mathfrak{v}_{0}$. Hence ad $X$ is represented by a triangular matrix with respect to the canonical basis

$$
\left\{T, Y_{1}, X_{2}, Y_{2}, \ldots, X_{n-1}, Y_{n-1}, Z_{0}\right\}
$$

of $\mathfrak{s}(\theta)$. Therefore all eigenvalues of ad $X$ are real, which completes the proof.

From now on, we shall study whether $(\mathfrak{s}(\theta),\langle\rangle$,$) is an algebraic Ricci soliton.$ We separate this into three cases, namely $\theta=\pi / 2, \theta \in] 0, \pi / 2[$, and $\theta=0$, and discuss them individually.

Firstly, we see that $(\mathfrak{s}(\pi / 2),\langle\rangle$,$) is always an algebraic Ricci soliton.$

Proposition 4.2. Let $n \geq 2$. Then $(\mathfrak{s}(\pi / 2),\langle\rangle$,$) is an algebraic Ricci soliton.$ 
Proof. We consider the basis $\left\{X_{1}, Y_{1}, \ldots, X_{n-1}, Y_{n-1}, Z_{0}\right\}$ of $\mathfrak{s}(\pi / 2)$. Denote by Ric the matrix expression of the Ricci operator of $\mathfrak{s}(\pi / 2)$ with respect to this basis. Then Proposition 3.6 yields that

$$
\operatorname{Ric}=\frac{1}{2}\left(\begin{array}{cccc}
-1 & & & \\
& \ddots & & \\
& & -1 & \\
& & & n-1
\end{array}\right) .
$$

In order to show that it is an algebraic Ricci soliton, we define

$$
c:=-\frac{n+1}{2}, \quad D:=\frac{n}{2}\left(\begin{array}{cccc}
1 & & & \\
& \ddots & & \\
& & 1 & \\
& & & 2
\end{array}\right) .
$$

Then one can show directly that $\operatorname{Ric}=c \cdot \mathrm{id}+D$ and $D \in \operatorname{Der}(\mathfrak{s}(\pi / 2))$.

Remark 4.3. Note that it has already been known from [12] that $(\mathfrak{s}(\pi / 2),\langle\rangle$, is an algebraic Ricci soliton. In fact, $\mathfrak{s}(\pi / 2)$ is the Heisenberg Lie algebra, and $\langle$,$\rangle is the standard inner product.$

Secondly, we consider the case $\theta \in] 0, \pi / 2[$. In this case, $(\mathfrak{s}(\theta),\langle\rangle$,$) cannot$ be an algebraic Ricci soliton. The proof starts with the following lemma for a derivation of $\mathfrak{s}(\theta)$.

Lemma 4.4. Let $n \geq 2$. If $\theta \in\left[0, \pi / 2\left[\right.\right.$, then $\left\langle D\left(Z_{0}\right), Y_{1}\right\rangle=0$ holds for any $D \in \operatorname{Der}(\mathfrak{s}(\theta))$.

Proof. Take any $D \in \operatorname{Der}(\mathfrak{s}(\theta))$. By the definition of a derivation, one has

$$
\left\langle D\left[T, Z_{0}\right], Y_{1}\right\rangle=\left\langle\left[D(T), Z_{0}\right], Y_{1}\right\rangle+\left\langle\left[T, D\left(Z_{0}\right)\right], Y_{1}\right\rangle .
$$

We calculate the both sides of this equation. By Lemma 3.5, the left-hand side satisfies

$$
\left\langle D\left[T, Z_{0}\right], Y_{1}\right\rangle=\left\langle D\left(\cos (\theta) Z_{0}\right), Y_{1}\right\rangle=\cos (\theta)\left\langle D\left(Z_{0}\right), Y_{1}\right\rangle .
$$

Next we calculate the right-hand side. It also follows from Lemma 3.5 that

$$
\left[\mathfrak{s}(\theta), Z_{0}\right] \subset \operatorname{span}\left\{Z_{0}\right\} .
$$

Then the first term of the right-hand side of (4.6) is

$$
\left\langle\left[D(T), Z_{0}\right], Y_{1}\right\rangle=0 .
$$

In order to calculate the second term, take the canonical orthonormal basis

$$
\left\{E_{i}\right\}=\left\{T, Y_{1}, X_{2}, Y_{2} \ldots, X_{n-1}, Y_{n-1}, Z_{0}\right\}
$$

of $\mathfrak{s}(\theta)$. Lemma 3.5 yields that

$$
\left\langle\left[T, E_{i}\right], Y_{1}\right\rangle= \begin{cases}(1 / 2) \cos (\theta) & \left(E_{i}=Y_{1}\right), \\ 0 & \text { (otherwise) } .\end{cases}
$$


Then the second term of the right-hand side of (4.6) satisfies

$$
\begin{aligned}
\left\langle\left[T, D\left(Z_{0}\right)\right], Y_{1}\right\rangle & =\left\langle\left[T, \sum\left\langle D\left(Z_{0}\right), E_{i}\right\rangle E_{i}\right], Y_{1}\right\rangle \\
& =\sum\left\langle D\left(Z_{0}\right), E_{i}\right\rangle\left\langle\left[T, E_{i}\right], Y_{1}\right\rangle \\
& =(1 / 2) \cos (\theta)\left\langle D\left(Z_{0}\right), Y_{1}\right\rangle .
\end{aligned}
$$

Altogether, we obtain

$$
\cos (\theta)\left\langle D\left(Z_{0}\right), Y_{1}\right\rangle=0+(1 / 2) \cos (\theta)\left\langle D\left(Z_{0}\right), Y_{1}\right\rangle .
$$

Since $\theta \in[0, \pi / 2[$, this completes the proof.

Proposition 4.5. Let $n \geq 2$. If $\theta \in] 0, \pi / 2[$, then $(\mathfrak{s}(\theta),\langle\rangle$,$) is not an algebraic$ Ricci soliton.

Proof. We show this by contradiction. Assume that $(\mathfrak{s}(\theta),\langle\rangle$,$) is an algebraic$ Ricci soliton. By definition, there exist $c \in \mathbb{R}$ and $D \in \operatorname{Der}(\mathfrak{s}(\theta))$ such that

$$
\operatorname{Ric}=c \cdot \mathrm{id}+D .
$$

One thus has

$$
\left\langle\operatorname{Ric}\left(Z_{0}\right), Y_{1}\right\rangle=c\left\langle Z_{0}, Y_{1}\right\rangle+\left\langle D\left(Z_{0}\right), Y_{1}\right\rangle .
$$

We calculate the both sides of this equation. By Proposition 3.6, we have

$$
\left\langle\operatorname{Ric}\left(Z_{0}\right), Y_{1}\right\rangle=(n / 2) \sin (\theta) \cos (\theta) .
$$

On the other hand, Lemma 4.4 yields that

$$
c\left\langle Z_{0}, Y_{1}\right\rangle+\left\langle D\left(Z_{0}\right), Y_{1}\right\rangle=0 .
$$

Since $\theta \in] 0, \pi / 2[$, this is a contradiction.

Lastly, we study the case $\theta=0$. First of all, we consider the case $n>2$, and show that $(\mathfrak{s}(0),\langle\rangle$,$) is not an algebraic Ricci soliton. As in the previous case,$ we start with the next lemma on a derivation. Note that $\mathfrak{v}_{0} \neq\{0\}$ if $n>2$.

Lemma 4.6. Assume that $n>2$, and let $D \in \operatorname{Der}(\mathfrak{s}(0))$. Then we have

(1) $\left\langle D\left(A_{0}\right), A_{0}\right\rangle=0$, and

(2) $\left\langle D\left(X_{k}\right), X_{k}\right\rangle+\left\langle D\left(Y_{k}\right), Y_{k}\right\rangle=\left\langle D\left(Z_{0}\right), Z_{0}\right\rangle$ for $k=2, \ldots, n-1$.

Proof. Take any $D \in \operatorname{Der}(\mathfrak{s}(0))$. The proof is similar to that of Lemma 4.4. We use the canonical orthonormal basis of $\mathfrak{s}(0)$,

$$
\left\{E_{i}\right\}=\left\{A_{0}, Y_{1}, X_{2}, Y_{2}, \ldots, X_{n-1}, Y_{n-1}, Z_{0}\right\}
$$

We show (1). By the definition of a derivation, one has

$$
\left\langle D\left[A_{0}, Z_{0}\right], Z_{0}\right\rangle=\left\langle\left[D\left(A_{0}\right), Z_{0}\right], Z_{0}\right\rangle+\left\langle\left[A_{0}, D\left(Z_{0}\right)\right], Z_{0}\right\rangle .
$$

The left-hand side of (4.19) satisfies

$$
\left\langle D\left[A_{0}, Z_{0}\right], Z_{0}\right\rangle=\left\langle D\left(Z_{0}\right), Z_{0}\right\rangle .
$$

In order to calculate the right-hand side, one needs

$$
\left\langle\left[E_{i}, Z_{0}\right], Z_{0}\right\rangle= \begin{cases}1 & \left(E_{i}=A_{0}\right), \\ 0 & \text { (otherwise). }\end{cases}
$$


Hence we have

$$
\left\langle\left[D\left(A_{0}\right), Z_{0}\right], Z_{0}\right\rangle=\sum\left\langle D\left(A_{0}\right), E_{i}\right\rangle\left\langle\left[E_{i}, Z_{0}\right], Z_{0}\right\rangle=\left\langle D\left(A_{0}\right), A_{0}\right\rangle .
$$

Similarly, one can see that

$$
\left\langle\left[A_{0}, E_{i}\right], Z_{0}\right\rangle= \begin{cases}1 & \left(E_{i}=Z_{0}\right) \\ 0 & \text { (otherwise) } .\end{cases}
$$

This yields that

$$
\left\langle\left[T, D\left(Z_{0}\right)\right], Z_{0}\right\rangle=\sum\left\langle D\left(Z_{0}\right), E_{i}\right\rangle\left\langle\left[T, E_{i}\right], Z_{0}\right\rangle=\left\langle D\left(Z_{0}\right), Z_{0}\right\rangle .
$$

Altogether, we have

$$
\left\langle D\left(Z_{0}\right), Z_{0}\right\rangle=\left\langle D\left(A_{0}\right), A_{0}\right\rangle+\left\langle D\left(Z_{0}\right), Z_{0}\right\rangle .
$$

This completes the proof of the first assertion.

We show (2). Take any $k=2, \ldots, n-1$. We start from

$$
\left\langle D\left[X_{k}, Y_{k}\right], Z_{0}\right\rangle=\left\langle\left[D\left(X_{k}\right), Y_{k}\right], Z_{0}\right\rangle+\left\langle\left[X_{k}, D\left(Y_{k}\right)\right], Z_{0}\right\rangle .
$$

One can calculate each term as follows:

$$
\begin{aligned}
\left\langle D\left[X_{k}, Y_{k}\right], Z_{0}\right\rangle & =\left\langle D\left(Z_{0}\right), Z_{0}\right\rangle, \\
\left\langle\left[D\left(X_{k}\right), Y_{k}\right], Z_{0}\right\rangle & =\sum\left\langle D\left(X_{k}\right), E_{i}\right\rangle\left\langle\left[E_{i}, Y_{k}\right], Z_{0}\right\rangle=\left\langle D\left(X_{k}\right), X_{k}\right\rangle, \\
\left\langle\left[X_{k}, D\left(Y_{k}\right)\right], Z_{0}\right\rangle & =\sum\left\langle D\left(Y_{k}\right), E_{i}\right\rangle\left\langle\left[X_{k}, E_{i}\right], Z_{0}\right\rangle=\left\langle D\left(Y_{k}\right), Y_{k}\right\rangle .
\end{aligned}
$$

This completes the proof of the second assertion.

Proposition 4.7. Let $n>2$. Then $(\mathfrak{s}(0),\langle\rangle$,$) is not an algebraic Ricci soliton.$

Proof. We show this by contradiction. Assume that $(\mathfrak{s}(0),\langle\rangle$,$) is an algebraic$ Ricci soliton. By definition, there exist $c \in \mathbb{R}$ and $D \in \operatorname{Der}(\mathfrak{s}(0))$ such that

$$
\text { Ric }=c \cdot \mathrm{id}+D \text {. }
$$

Then, for any $E \in \mathfrak{s}(0)$ with $\langle E, E\rangle=1$, one has

$$
\langle\operatorname{Ric}(E), E\rangle=\langle c E+D(E), E\rangle=c+\langle D(E), E\rangle \text {. }
$$

Let $V \in \mathfrak{v}_{0}$ be a unit vector, and we substitute $A_{0}, V$, and $Z_{0}$ for $E$. Then, Proposition 3.6 yields that

$$
\begin{aligned}
c+\left\langle D\left(A_{0}\right), A_{0}\right\rangle & =\left\langle\operatorname{Ric}\left(A_{0}\right), A_{0}\right\rangle=-(2 n+1) / 4, \\
c+\langle D(V), V\rangle & =\langle\operatorname{Ric}(V), V\rangle=-(2 n+1) / 4, \\
c+\left\langle D\left(Z_{0}\right), Z_{0}\right\rangle & =\left\langle\operatorname{Ric}\left(Z_{0}\right), Z_{0}\right\rangle=-(n+1) / 2 .
\end{aligned}
$$

These equations yield that

$$
\left\langle D\left(A_{0}\right), A_{0}\right\rangle=\langle D(V), V\rangle \neq\left\langle D\left(Z_{0}\right), Z_{0}\right\rangle .
$$

Lemma 4.6 (11) yields that

$$
0=\left\langle D\left(A_{0}\right), A_{0}\right\rangle=\langle D(V), V\rangle .
$$

Since $n>2$, we have $X_{2}, Y_{2} \in \mathfrak{v}_{0}$, which can be substituted for $V$. Thus, owing to Lemma 4.6 (2), we have

$$
\left\langle D\left(Z_{0}\right), Z_{0}\right\rangle=\left\langle D\left(X_{2}\right), X_{2}\right\rangle+\left\langle D\left(Y_{2}\right), Y_{2}\right\rangle=0 .
$$

This is a contradiction. 
If $n=2$, however, $(\mathfrak{s}(0),\langle\rangle$,$) is indeed an algebraic Ricci soliton.$

Proposition 4.8. Let $n=2$. Then $(\mathfrak{s}(0),\langle\rangle$,$) is an algebraic Ricci soliton.$

Proof. Denote by Ric the matrix expression of the Ricci operator with respect to the basis $\left\{A_{0}, Y_{1}, Z_{0}\right\}$ of $\mathfrak{s}(0)$. Then, Proposition 3.6 yields that

$$
\operatorname{Ric}=\left(\begin{array}{ccc}
-5 / 4 & & \\
& -3 / 4 & \\
& & -3 / 2
\end{array}\right) .
$$

Let us define

$$
c:=-\frac{5}{4}, \quad D:=\left(\begin{array}{lll}
0 & & \\
& 1 / 2 & \\
& & -1 / 4
\end{array}\right) .
$$

Then we can show directly that $\operatorname{Ric}=c \cdot \mathrm{id}+D$, and $D \in \operatorname{Der}(\mathfrak{s}(0))$.

Remark 4.9. It is known that the Lie hypersurface $(\mathfrak{s}(0),\langle\rangle$,$) is an algebraic$ Ricci soliton when $n=2$. In fact, Lauret ([13]) has classified three-dimensional algebraic Ricci solitons on solvable Lie algebras. Among them, there are Lie algebras $\mathfrak{r}_{\alpha}=\operatorname{span}\{A, X, Y\}$, for $\alpha \in[-1,1]$, whose bracket relations are given by

$$
[A, X]=X, \quad[A, Y]=\alpha Y .
$$

Let $\langle,\rangle_{0}$ be the inner product so that the basis $\{A, X, Y\}$ is orthonormal. Then $\left(\mathfrak{r}_{\alpha},\langle,\rangle_{0}\right)$ is an algebraic Ricci soliton $([13])$. Note that our Lie hypersurface $(\mathfrak{s}(0),\langle\rangle$,$) is obviously isometric to \left(\mathfrak{r}_{1 / 2},\langle,\rangle_{0}\right)$.

In consequence of the arguments above we conclude the following theorem.

Theorem 4.10. The Lie hypersurfaces $S(\theta)$.o in $\mathbb{C H}^{n}$ is a Ricci soliton if and only if

(1) $\theta=\pi / 2$, or

(2) $n=2$ and $\theta=0$.

\section{REFERENCES}

[1] J. Berndt, Homogeneous hypersurfaces in hyperbolic spaces, Math. Z., 229 (1998), 589600.

[2] J. Berndt \& M. Brück, Cohomogeneity one actions on hyperbolic spaces, J. Reine Angew. Math., 541 (2001), 209-235.

[3] J. Berndt, J. C. Díaz-Ramos \& H. Tamaru, Hyperpolar Homogeneous foliations on symmetric spaces of noncompact type, J. Differential Geom. 86, 191-235 (2010)

[4] J. Berndt \& H. Tamaru, Homogeneous codimension one foliations on noncompact type symmetric spaces, J. Differential Geom., 63 (2003), no. 1, 1-40.

[5] J. Berndt \& H. Tamaru, Cohomogeneity one actions on noncompact symmetric spaces with a totally geodesic singular orbit, Tohoku Math. J., 56 (2004), no. 2, 163-177.

[6] J. Berndt \& H. Tamaru, Cohomogeneity one actions on noncompact symmetric spaces of rank one, Trans. Amer. Math. Soc., 359 (2007), no. 7, 3425-3438.

[7] J. T. Cho \& M. Kimura, Ricci solitons of compact real hypersurfaces in Kähler manifolds, Math. Nachricten, 284 (2011), 1385-1393.

[8] J. C. Díaz-Ramos, M. Domínguez-Vázquez \& A. Kollross, Polar actions on complex hyperbolic spaces, preprint, arXiv:1208.2823v2. 
[9] T. Hamada, Y. Hoshikawa \& H. Tamaru, Curvatures properties of Lie hypersurfaces in the complex hyperbolic space, J. Geom., 103 (2012), no. 2, 247-261.

[10] A. Kubo \& H. Tamaru, A sufficient condition for congruency of orbits of Lie groups and some applications, Geom. Dedicata, to appear, arXiv:1212.3893v1.

[11] C. Gorodski \& N. Gusevskii, Complete minimal hypersurfaces in complex hyperbolic space, manuscripta math., 103 (2000), 221-240.

[12] J. Lauret, Ricci soliton homogeneous nilmanifolds, Math. Ann., 319 (2001), 715-733.

[13] J. Lauret, Ricci soliton solvmanifolds, J. reine angew. Math., 650 (2011), 1-21.

[14] M. Lohnherr \& H. Reckziegel, On ruled real hypersurfaces in complex space forms, Geom. Dedicata, 74 (1999), 267-286.

[15] P. Ryan, Intrinsic properties of real hypersurfaces in complex space forms, in: Geometry and topology of submanifolds, X, (Beijing/Berlin, 1999), 266-273, World Sci. Publ., River Edge, NJ, 2000.

[16] H. Tamaru, Parabolic subgroups of semisimple Lie groups and Einstein solvmanifolds, Math. Ann., 351 (2011), 51-66

(T. Hashinaga) Department of Mathematics, Hiroshima University, Higashi-Hiroshima 739-8526, JAPAN

(A. Kubo) Department of Mathematics, Hiroshima University, Higashi-Hiroshima 739-8526, JAPAN

(H. Tamaru) Department of Mathematics, Hiroshima University, Higashi-Hiroshima 739-8526, JAPAN

E-mail address, A. Kubo: akira-kubo@hiroshima-u.ac.jp

E-mail address, T. Hashinaga: hashinaga@iroshima-u.ac.jp

E-mail address, H. Tamaru: tamaru@math.sci.hiroshima-u.ac.jp 